\title{
Entre mémoire et preuve : le rôle de l'écrit dans les exploitations agricoles
}

\author{
Armelle Mazéa, ${ }^{a}$, Marianne Cerf $^{b}$, Marianne Le Bail ${ }^{c}$, François Papy ${ }^{d}$ \\ a Économiste, INRA, UMR SAD-APT, INA P-G, 16 rue Claude Bernard, 75231 Paris cedex 05, France \\ b Ergonome, INRA, UMR SAD-APT, INA P-G, 16 rue Claude Bernard, 75231 Paris cedex 05, France \\ c Agronome, INA, UMR SAD-APT, INA P-G, 16 rue Claude Bernard, 75231 Paris cedex 05, France \\ d Agronome, INRA, UMR SAD-APT, INA P-G, 16 rue Claude Bernard, 75231 Paris cedex 05, France
}

\begin{abstract}
Dans tout acte de production, l'écrit fait partie d'un travail de mémorisation pour constituer des repères en vue d'organiser et de planifier une activité ultérieure. De par sa complexité, l'agriculture n'y échappe pas. Les traces qui en résultent intéressent les firmes dans leur quête d'une transparence préventive. Il s'ensuit un décalage entre des écritures «spontanées » au sein de l'exploitation et la nécessité pour les firmes de formater des informations d'une grande diversité. Deux aspects de cette question nous intéressent. D'une part, voir en quoi l'objectif de traçabilité suppose de nombreux ajustements qui interfèrent avec les savoir-faire et réévaluent les activités de production. De l'autre, ouvrir un débat sur la construction de la preuve dont on voit bien ici qu'elle relève tout autant de la confiance que garantit l'engagement de l'agriculteur que de l'irréfutabilité associée à l'écrit.
\end{abstract}

La Rédaction

\section{Mots-clés :}

écrit ; mémorisation ; organisation; exploitation agricole; information ; traçabilité

\section{Keywords:} writings; memorization; organisation; farm management; information management; traceability
Résumé - La société manifeste actuellement des exigences de traçabilité vis-à-vis de l'agriculture et considère que pour y répondre, l'écrit peut, sous certaines conditions, avoir un statut de preuve. Ceci nous a conduits à analyser la production de documents écrits dans l'exploitation agricole. Nous avons entrepris une analyse des pratiques de saisie et de stockage des informations relatives au processus de production sur un échantillon d'une dizaine d'exploitations agricoles de grande culture en Picardie, en mettant l'accent sur le rôle de l'écrit comme aide cognitive et support de mémorisation pour les agriculteurs. Nous mettons ainsi en évidence une diversité de formats de saisie et de reports successifs sur différents supports qu'il est possible de relier à la diversité des modes d'organisation et de gestion interne des exploitations. Pour concilier le rôle de mémoire de l'écrit dans la gestion des exploitations et celui de preuve pour les clients de l'agriculture, nous suggérons plusieurs pistes.

\begin{abstract}
From memory to proof : the role of writings on farm. Nowadays, the society requires more traceability of production practices and to achieve this goal, assigns sometimes to writings the status of a proof. Therefore, we studied the documentation produced by farmers. Based on detailed inquiries about farmers' writings practices on a sample of ten crop farms in Northern France, the analysis focuses on written data on the production process and their role as a cognitive support for memorization for the farmers. We show a diversity of writings according to the type of format created by farmers, and the successive postings of information. The data collected suggest that this diversity is less connected to the various levels of proof requested by agro-food firms than to the way farmers organize and manage their productive activity. According to this result, alternative solutions are proposed to reduce the discrepancy between the role of the writings as memorization support on farms and their role as proof for farmers' customers.
\end{abstract}

Suite aux crises sanitaires qui ont marqué l'Europe au cours des années 1990, les agriculteurs sont confrontés à une demande toujours croissante d'informations sur leurs façons de produire. Cela se traduit notamment

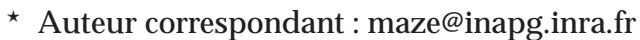

par la floraison de cahiers des charges définis tant par la grande distribution (secteur de la viande en particulier) que par les industries agro-alimentaires, voire les organismes de collecte (céréales notamment). Les débats récents sur l'agriculture raisonnée s'inscrivent également 
dans cette logique d'une transparence accrue sur les modes de production (Paillotin, 2000 ; Mazé et al., 2002). Ainsi, il semblerait que la chose soit entendue : les agriculteurs devraient formaliser leurs façons de produire pour répondre aux attentes de la société en matière de traçabilité et de respect de l'environnement.

La multiplication des demandes et l'absence de concertation entre les interlocuteurs des agriculteurs sur les informations demandées et leurs supports, conduisent les agriculteurs à remplir de plus en plus de formulaires écrits appelés à constituer des preuves sur les façons de produire. Divers travaux sont conduits avec pour objectif d'optimiser les flux d'informations entre les agriculteurs et leurs interlocuteurs, et les solutions proposées aux agriculteurs privilégient aujourd'hui l'informatisation des transferts d'informations, comme cela est souvent réalisé dans les travaux en économie et gestion sur la conception de systèmes d'information dans des organisations complexes (Aoki, 1986 ; Radner, 1993; Reix, 1996).

De telles approches écartent les questions relatives à la collecte initiale des informations et à son coût (Barzel, 1977) et tendent à faire abstraction des pratiques des acteurs en matière d'écrit et d'utilisation des informations. Or les agriculteurs notent pour eux-mêmes des informations. Qui n'a pas entendu parler de l'agenda, du carnet de plaine de l'agriculteur? Mais que contient-il ? Pourquoi l'agriculteur éprouve-t-il le besoin de noter certaines informations? N. Joly (1997) montre que les agriculteurs consignent des informations sur leur agenda pour accroître leur maîtrise symbolique et pratique de leur activité. D'autres écrits existent dans l'exploitation. Dans quelle mesure l'ensemble des écrits produits par l'agriculteur pour accroître la maîtrise de son activité peuventils être utilisés, à moindre coût, comme preuve du respect des cahiers des charges des clients?

Pour aborder cette question, nous nous sommes interrogés sur les divers documents construits par l'agriculteur pour saisir des informations relatives à l'organisation et la conduite de ses productions. Nous avons caractérisé ces documents selon leur type de support (exemples : fiche, carnet, fichier informatique, mais aussi support primaire ou secondaire selon l'enchaînement des saisies des informations), selon la structure que l'agriculteur donne à l'information écrite et que nous appelons format (exemples : structuration de l'information par date, par ouvrier, par parcelle, sous forme de programme d'action, de carte... ), selon le contenu, en nous attachant plus particulièrement au degré de précision des informations notées. Plus qu'aux échanges d'informations entre agriculteurs et firmes agro-alimentaires, nous nous sommes particulièrement intéressés à la compatibilité des formats construits par les uns, demandés par les autres, car ils traduisent leur manière de structurer les informations ${ }^{1}$.

\section{L'écrit dans l'organisation}

Plutôt que de suivre la voie ouverte par les sociologues (Fraenkel, 1995; Grosjean et Lacoste, 1998; Cochoy, et al. 1998) qui se sont surtout penchés sur la façon dont les acteurs utilisent leurs écrits dans les rapports de travail et de pouvoir existant dans l'entreprise, nous proposons dans cet article de comprendre comment les écrits produits par les agriculteurs sont reliés à leurs choix organisationnels et contractuels, en nous appuyant sur certains développements récents des théories des organisations. Dans le cas de l'exploitation agricole, les travaux de Maxime et al. (1997), d'Aubry et al. (1998) ont montré que son organisation est construite et portée par l'agriculteur qui articule, dans l'espace et le temps, les formes d'utilisation des ressources disponibles ou susceptibles de l'être, et des contraintes externes, en particulier les exigences contenues dans les contrats. Mais ces auteurs n'ont pas analysé comment les agriculteurs élaborent cette construction dans laquelle nous pensons que l'écrit a une place importante.

C'est donc dans cette perspective que nous étudions les écrits que l'agriculteur produit pour lui-même avant de nous intéresser à ceux qui interviennent dans les échanges avec leurs clients. En accord avec les travaux de N. Joly (op. cit.), nous faisons l'hypothèse que les écrits construits par l'agriculteur constituent une mémoire externe utile pour la maîtrise de ses activités productives et jouent le rôle d'une aide cognitive pour l'agriculteur. Les écrits reflètent ce que Fabbe-Costes (1997) appelle une mémorisation volontaire où l'acteur note ce qu'il ne veut pas oublier, se construisant ainsi son propre système de mémorisation en opérant un tri conscient dans les informations qu'il lui semble utile de conserver. Comme le souligne cet auteur, les écrits sont à distinguer d'une mémorisation spontanée, ancrée dans l'expérience du quotidien. On peut donc penser que toutes les informations utiles à l'activité productive ne sont pas consignées par écrit dans la mesure où la conscience qu'a l'agriculteur de son organisation lui permet de les retrouver.

De fait, comme l'a montré Lorenz (2001) dans d'autres types d'entreprises, les informations sont pour partie inscrites dans l'organisation et les dispositifs matériels élaborés par les acteurs et ne nécessitent pas d'être mémorisées sous une autre forme. Ainsi, nous considérons que

\footnotetext{
1 Cette étude s'inscrit dans le cadre d'un programme de recherche-intervention pluridisciplinaire engagé depuis 1997 sur le thème de la certification des exploitations agricoles et mené en partenariat entre Agro-Transfert et les chambres d'agriculture de Picardie. Cette recherche a reçu le soutien financier du conseil régional de Picardie.
} 
les écrits qui servent à l'activité productive de l'exploitation, les processus de mémorisation des informations pour réaliser cette activité et l'organisation des moyens de production sont étroitement liés.

Mais, comme le souligne Fabbe-Costes (op. cit.), l'existence d'un système de mémorisation propre à un acteur, ici l'agriculteur, ne signifie pas que les informations qu'il contient soient accessibles à d'autres acteurs, par exemple ses clients. Au sein des grandes entreprises, l'accessibilité, par l'ensemble des acteurs, des informations propres à chacun repose sur une mémoire organisationnelle constituée par l'enregistrement et la distribution des connaissances produites pendant l'activité des acteurs (Owen, 1986; Ackerman et Malone, 1990). Mais encore faut-il que les acteurs aient intérêt à mettre en commun leurs connaissances : peut-on considérer que les agriculteurs et leurs interlocuteurs des firmes agro-alimentaires souhaitent cette mise en commun? Sans entrer dans ce débat, nous nous interrogerons ici sur la compatibilité des formats élaborés par les agriculteurs et ceux construits par leurs interlocuteurs. A priori, cette compatibilité ne va pas de soi.

Notre étude sur les pratiques des agriculteurs en matière de gestion des informations sur leur manière de produire cherche à étayer les deux hypothèses suivantes : (i) les informations que les agriculteurs souhaitent mémoriser sont pour partie formalisées par écrit, et pour partie inscrites dans l'organisation; (ii) les formats de saisie et de stockage des informations dépendent des choix organisationnels des agriculteurs, et de leur traduction dans l'activité productive (planification, contrôle du travail, contrôle des quantités apportées, pilotage des cultures...). Si ces deux hypothèses sont bien vérifiées, alors, il paraît logique de constater un décalage entre les documents produits par les agriculteurs et ceux demandés par leurs partenaires économiques (firmes, coopératives).

\section{Méthodes}

Nous avons fait des enquêtes dans dix exploitations choisies de manière à diversifier leur insertion dans des réseaux de relations techniques (avec les organismes de développement, avec les firmes) et la nature des débouchés pour leurs productions. Il s'agit essentiellement d'exploitations de grande culture, mais une des exploitations enquêtées a aussi des vaches laitières, une autre a un élevage de truies. Six exploitations, situées dans le Santerre, produisent principalement des légumes transformés et des pommes de terre; quatre, sur le Plateau picard sud, ont une orientation céréalière dominante. Il s'agit d'exploitations de superficie généralement au-dessus de la moyenne picarde (78 ha en 1997), dans lesquelles le travail est réalisé par plusieurs personnes. Nous avons considéré que ce dernier point pouvait être important pour le développement d'écrits internes à l'exploitation.

Notre travail repose avant tout sur des entretiens semi-directifs conduits auprès des agriculteurs pour reconstituer a posteriori l'organisation des assolements et des techniques (voir Papy (2001) et Ognov (2000, 2001) pour la méthode), inventorier les documents qu'ils produisent et appréhender la manière dont ils collectent, stockent, et transmettent les informations. Nous avons également collecté, dans la mesure du possible, les documents produits (copie de pages d'agenda, sorties informatiques, fiche par parcelle culturale ou par opération, document d'assolement, etc.). Par ailleurs, des entretiens semi-directifs auprès des entreprises agro-alimentaires de la région ont été menés pour identifier les documents qu'ils demandent à leurs fournisseurs et la façon dont ils en tiennent compte pour leur gestion interne et leurs échanges avec les agriculteurs.

Le traitement des données a consisté, d'abord, à identifier et caractériser l'ensemble des documents que les agriculteurs génèrent, que ce soit pour leur propre gestion technique ou technico-économique ou pour les besoins de traçabilité exigés par leurs clients. Pour discuter des relations entre la diversité des documents mobilisés par les agriculteurs et les caractéristiques des choix organisationnels et contractuels propres à chaque exploitation, plusieurs indicateurs synthétiques sur l'organisation des exploitations agricoles ont été élaborés par rapport à leurs effets possibles sur la quantité d'information à gérer par l'agriculteur pour ses conduites techniques (Tab. 1). La complexité des assolements est évaluée en tenant compte de la régularité des rotations (Maxime et al., $o p$. cit.) et du morcellement du parcellaire évalué à partir de trois critères : la taille, le nombre de parcelles et la division de l'exploitation en blocs géographiquement séparés. L'intensité de la pression externe est évaluée en fonction du nombre de clients, de la stabilité des relations commerciales de l'agriculteur et de l'importance des productions devant répondre à des cahiers des charges relativement contraignants en termes de suivi et de transmission d'informations.

Nous avons cherché à interpréter les résultats en considérant que le nombre des espèces cultivées, la multiplication de petites parcelles, surtout si elles sont disposées en blocs éloignés les uns des autres, rendaient complexe l'organisation des assolements et des successions de culture. De même nous avons estimé que la difficulté de répondre à toutes les exigences de traçabilité s'accroissent avec la multiplication du nombre de clients et la part de légumes de conserve et de pommes de terre dans l'assolement. Ces choix organisationnels et contractuels sont déterminés par l'agriculteur en fonction du degré de flexibilité que ce dernier souhaite se donner par rapport à sa stratégie commerciale et sa capacité à répondre à d'éventuelles opportunités de marché. 
Tableau 1. Choix organisationnels et contractuels.

\begin{tabular}{|l|l|}
\hline \multicolumn{2}{|l|}{ agriculteurs } \\
\hline Organisation des assolements \\
\hline $\begin{array}{l}\text { Stabilité de la } \\
\text { succession des } \\
\text { structures }\end{array}$ & $\begin{array}{l}\text { Rotation-type stable } \\
\text { Rotation-type assez stable } \\
\text { Pas de rotation stable }\end{array}$ \\
\hline $\begin{array}{l}\text { Nature du } \\
\text { Parcellaire }\end{array}$ & $\begin{array}{l}\text { Grandes parcelles }(+\mathrm{de} 10 \mathrm{ha}) \\
\text { Petites parcelles }\end{array}$ \\
\cline { 2 - 3 } & $\begin{array}{l}\text { Nb parcelles faible }(\mathrm{nb}<25 \text { parcelles }) \\
\text { Nb parcelles élevé }\end{array}$ \\
\hline Division de l'EA & blocs d'exploitation géographiquement éloignés \\
\hline
\end{tabular}

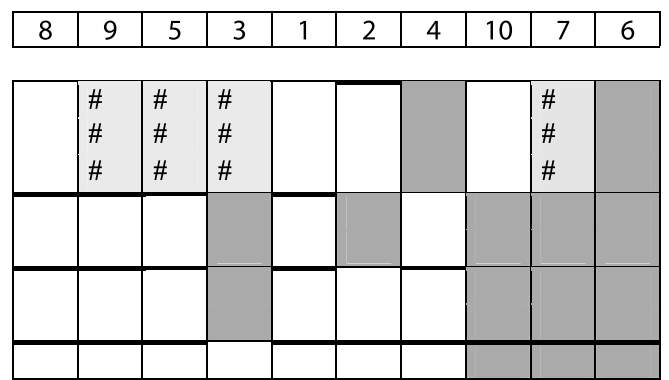

\section{Relations avec les clients}

\begin{tabular}{|l|l|l|}
\hline $\begin{array}{l}\text { Degré de } \\
\text { flexibilité } \\
\text { commerciale }\end{array}$ & $\begin{array}{l}\text { Forte stabilité revenus (quota betterave ou lait) et } \\
\text { peu d'autres clients } \\
\text { Stabilité avec }+60 \% \text { à la coopérative et nombre } \\
\text { de clients moyen (3-4) } \\
\text { Forte flexibilité commerciale (capacité stockage) }\end{array}$ & \\
\hline $\begin{array}{l}\text { Exigence en } \\
\text { tracabilité }\end{array}$ & $\begin{array}{l}\text { Production légume ou pomme de terre sous } \\
\text { contrat }\end{array}$ & \\
\hline
\end{tabular}

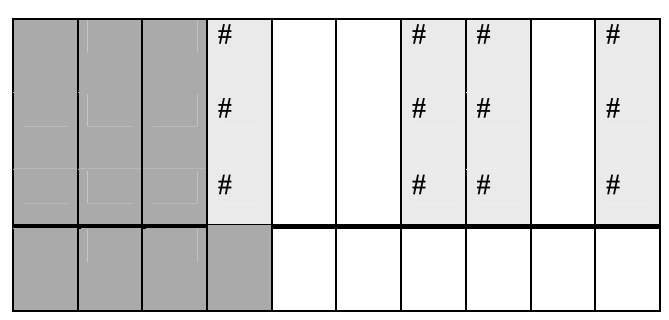

Classement des exploitations entre deux extrêmes : de fortes sollicitations des clients $(8,9,5)$ et de fortes contraintes d'exploitations $(7,6)$

\begin{tabular}{|l|l}
\hline$\#$ & $\begin{array}{l}\text { contrainte faible } \\
\text { contrainte moyenne } \\
\text { contrainte forte }\end{array}$
\end{tabular}

Tableau 2. Type de documents sur les processus de production recensés dans les exploitations enquêtées.

\begin{tabular}{|c|c|c|c|c|c|c|c|c|c|c|c|}
\hline & agriculteurs & 8 & 9 & 5 & 3 & 1 & 2 & 4 & 10 & 7 & 6 \\
\hline \multirow{4}{*}{ Document assolement } & Annuel & & & & & & & & & & \\
\hline & Pluriannuel & & & & & & & & & & \\
\hline & $\begin{array}{l}\text { Pluriannuel et prévisionnel } \\
\text { informatisé }\end{array}$ & & & & & & & & & & \\
\hline & Pluriannuel, prévisionnel, papier & & & & & & & & & & \\
\hline \multirow{2}{*}{$\begin{array}{l}\text { Document } \\
\text { prévisionnel phyto- } \\
\text { sanitaire }\end{array}$} & Par produit & & & & & & & & & & \\
\hline & Par produit puis par culture & & & & & & & & & & \\
\hline \multirow{2}{*}{$\begin{array}{l}\text { Document } \\
\text { prévisionnel fumure }\end{array}$} & Calcul des fumures & & & & & & & & & & \\
\hline & Apport/parcelle & & & & & & & & & & \\
\hline \multirow{4}{*}{ Carnet de plaine } & Agenda & & & & & & & & & & \\
\hline & Fiche par lot de parcelles & & & & & & & & & & \\
\hline & Fiche par parcelle & & & & & & & & & & \\
\hline & Fiche par opération & & & & & & & & & & \\
\hline Autres & & & & & & & & & & & \\
\hline
\end{tabular}

En grisé : présence du document sur l'exploitation

\section{Résultats}

L'analyse du système d'informations écrites dans les exploitations est décomposée en deux points : les formats utilisés pour la saisie et pour les reports ; le contenu des informations elles-mêmes. La diversité constatée est alors mise en relation avec la diversité des modes d'organisation interne des exploitations et de leurs relations avec leur environnement économique.

\section{La diversité des documents, des supports et formats}

Dans les dix exploitations que nous avons enquêtées un premier constat s'impose (Tab. 2) : il n'y a pas systématiquement présence des mêmes types de documents (Encadré 1). Si toutes les exploitations disposent $d^{\prime}$ 'un document d'assolement, d'un carnet de plaine, d'un document prévisionnel des achats phytosanitaires, peu 
Encadré 1. Glossaire des documents.

Document d'assolement : ce document fournit, pour une année donnée, la répartition des cultures sur les différentes parcelles de l'exploitation.

Carnet de plaine : carnet sur lequel l'agriculteur relève ce qu'il fait sur ses différentes cultures.

Document prévisionnel des achats phytosanitaires : ce document récapitule, à l'avance, la nature et les quantités de produits phytosanitaires dont l'agriculteur a besoin pour les acheter en gros et en morte-saison (c'est moins cher), quelques mois avant leur utilisation.

Programme prévisionnel de fumure : ce document récapitule, à l'avance, la nature et les quantités d'engrais dont l'agriculteur a besoin pour les acheter en gros et en morte-saison (c'est moins cher), quelques mois avant leur utilisation.

Fiche par parcelle : généralement, feuille recto verso comprenant par parcelle de culture le relevé synthétique des différentes étapes de la culture (dates, doses, noms des produits ou des machines... ) ; également appelée « fiche parcellaire».

disposent d'un programme prévisionnel de fumure, par exemple. La distinction entre exploitations est plus nette si l'on analyse le format des documents. Le plan d'assolement peut ainsi être annuel ou pluriannuel ou encore pluriannuel et prévisionnel.

Le papier reste le support de saisie, voire de stockage, dominant. Néanmoins, comme nous le verrons plus loin, certains agriculteurs reportent les informations du support papier vers un support informatique.

La comparaison des différents types de format, qui servent à recueillir les informations au fil du temps, fournit des éléments intéressants sur la diversité des finalités de recueil des informations (Tab. 2). Ainsi, alors qu'en majorité, les agriculteurs structurent les informations selon la date de réalisation du travail, d'autres les structurent par lots de parcelles (agriculteur $\mathrm{n}^{\circ} 10$ ), par parcelle (agriculteur $\mathrm{n}^{\circ} 4$ ) ou par opération (agriculteur $\mathrm{n}^{\circ} 2$. Une entrée par la date se traduit majoritairement par l'utilisation d'un agenda et reflète la volonté de contrôler l'avancement du travail. L'entrée par parcelle correspondrait à une préoccupation de suivi technique de la culture $\left(n^{\circ} 4\right)$, tandis que l'entrée par lot et par opération dénoterait une volonté d'organiser le travail ( ${ }^{\text {os }} 2$ et 10$)$. De même, l'existence d'un document annuel révèle le souci d'affecter la ressource "terre » aux différentes cultures de sorte à maintenir un équilibre entre elles, tandis que la conservation des informations sur plusieurs années, grâce à un format pluriannuel, témoigne du souci de gérer les successions de cultures en tenant compte des effets d'une culture sur la suivante et des délais de retour d'une culture sur elle-même. Dans une perspective de traçabilité, un tel format du document d'assolement peut permettre la reconstitution de l'historique des parcelles. Mais un même document présent dans plusieurs exploitations peut y remplir des fonctions différentes (Encadré 2).
Encadré 2. Le document d'assolement pluriannuel : un outil de gestion spatiale et temporelle.

Ce document est présent dans la plupart des exploitations enquêtées (7 sur 10). Il remplit différentes fonctions dans la gestion technique. Il est utilisé : (i) pour respecter des contraintes de succession de cultures, en particulier pour prévoir l'emplacement d'une culture comme la pomme de terre qui exige que soit respecté un délai de retour de la culture sur la même parcelle (exploitations 8 et 9) ou pour vérifier, a posteriori, le respect de ces délais de retour (exploitation 1); (ii) pour gérer des équilibres biophysiques dépassant un cycle annuel comme dans le cas des pratiques de fumure de fond (exploitation 3); (iii) pour simuler différents scénarios de revenus dans les années à venir (afin de négocier des emprunts, mettre en place d'autres activités (chambre d'hôte)) (exploitation 4). Enfin, dans certains cas, ce document peut servir de substitut à un enregistrement par parcelle pour les exploitations 5 et 8 alors qu'elles enregistrent peu d'informations sur leur agenda, seul autre document écrit pour la gestion technique des cultures. Les trois exploitations qui n'en ont pas $(6,7,10)$ ont des parcellaires très morcelés et dispersés (Tab. 2).

L'examen des pratiques de report d'un document à un autre montre que les fiches parcellaires demandées par beaucoup de clients, quand elles sont remplies (6 et 9 ne les remplissent que s'ils savent qu'elles leur seront demandées, ce qui n'est pas systématique en céréales et pommes de terre, par exemple), le sont a posteriori, à l'aide des documents que les agriculteurs ont élaborés pour leur propre gestion. Au-delà du report sur des fiches demandées par les clients, quatre agriculteurs sur dix reportent des informations depuis le support primaire vers d'autres supports pour un usage interne (Tab. 3).

Ces reports sont souvent l'occasion d'une modification de la structuration des informations (réorganisation selon un nouveau format) : ainsi pour les agriculteurs 1 , 2,3 et 9, le report se fait depuis un agenda structuré par la date, vers une fiche parcellaire (Fig. 1), qu'elle soit informatisée ou non. Si l'agriculteur 9 a deux agendas, c'est que le premier ne le quitte jamais, il y note en temps réel les opérations accomplies, tandis que le second permet le soir de faire la synthèse des heures de travail de la journée (les siennes et celles des ouvriers) et de noter de manière prévisionnelle les échéances importantes. Ces informations sont reprises les jours suivants par une autre personne qui saisit ces informations sur un support informatique.

\section{La diversité des informations saisies}

L'examen plus fin de ce qui est noté sur les supports primaires pour le suivi des productions (Tab. 4) montre que les agriculteurs ne notent pas les mêmes informations. Ne sont notées, dans la majorité des cas, que les informations qui servent à anticiper certains besoins (en 
Tableau 3. Les pratiques de report écrit internes à l'exploitation.

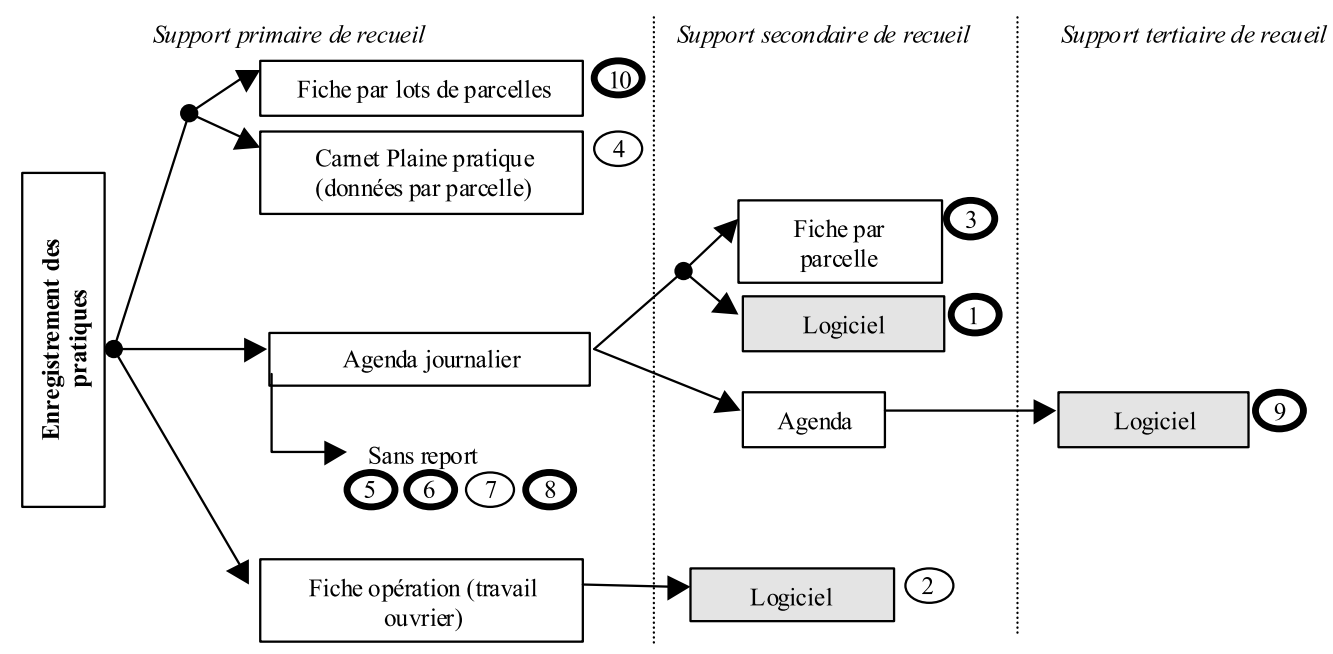

Les numéros renvoient au numéro d'exploitation, les cercles plus épais figurent les cas où des fiches sont à foumir aux clients

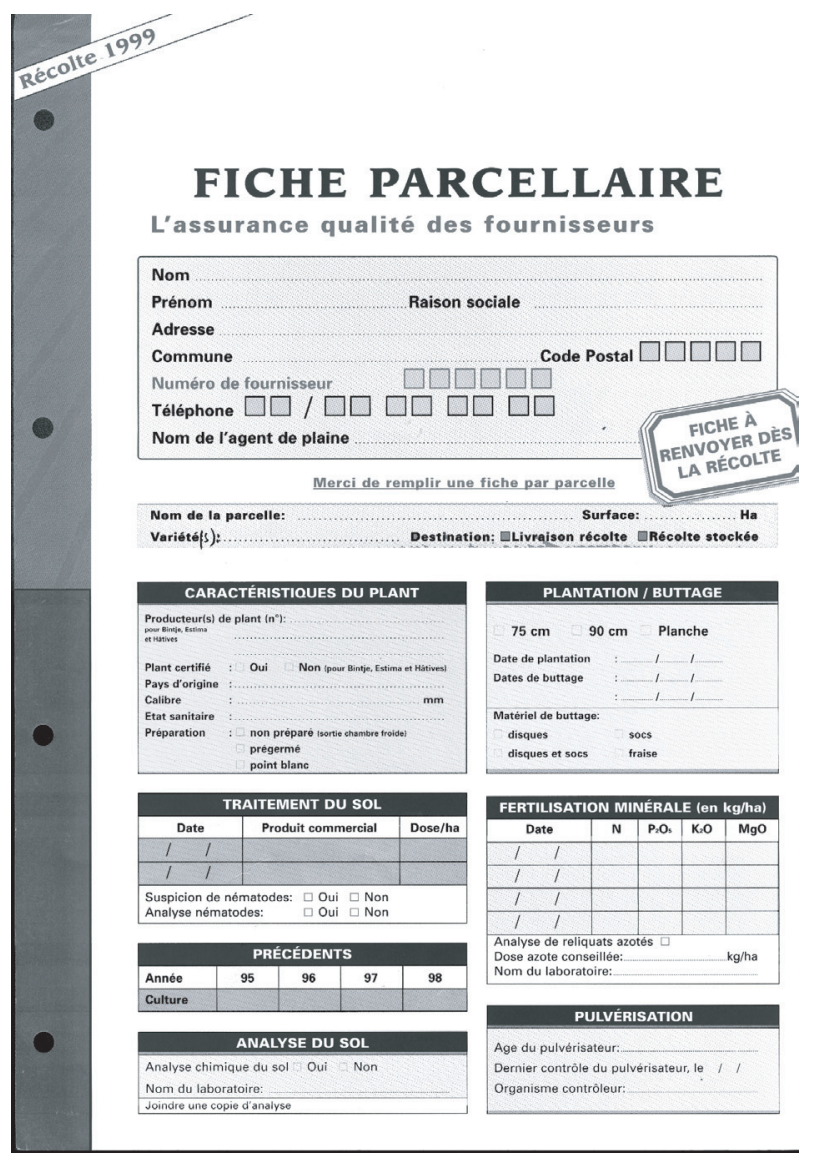

Fig. 1. Première page de fiche parcellaire proposée par un industriel aux agriculteurs qui l'approvisionnent en pommes de terre.

eau, en éléments fertilisants), à suivre la réalisation du travail ou celles qui pourront être réutilisées pour la planification des activités des années à venir : c'est particulièrement vrai pour les informations sur la dose et la nature des produits utilisés (Fig. 2). Ces informations seront aussi utiles pour les demandes des clients bien que ces informations soient rarement notées à la parcelle, échelle à laquelle sont demandées les informations par les clients, comme nous le verrons. Un agriculteur ( $\left.\mathrm{n}^{\circ} 9\right)$ note des éléments permettant de retrouver le travail fait par chaque ouvrier, et un autre des éléments susceptibles de guider le pilotage de la culture et d'être mobilisés dans le diagnostic ( $\left.n^{\circ} 10\right)$. Le fait que certains agriculteurs ( $\mathrm{n}^{\text {os }} 2$ à 7 ) notent, sur leur agenda ou leurs fiches par opération, les parcelles concernées indiquerait le souci de mémoriser l'organisation spatiale de la réalisation du travail.

Sauf dans un cas, la notation des informations n'est pas déléguée. Il semble que noter soit un moyen pour l'agriculteur de rester en contact avec le travail fait lorsqu'il ne l'exécute pas lui-même. Mais cela signifie aussi que ce qui est noté l'est généralement en fin de journée après un échange oral avec les autres personnes travaillant dans l'exploitation, ce qui peut engendrer des imprécisions ou des risques d'erreurs.

Quand l'information notée sur le support primaire comporte des lacunes, en regard de ce qui est demandé par les clients, par exemple, cela ne signifie pas que les informations manquantes ne sont pas mémorisées ailleurs que dans l'écrit. La preuve en est que lorsqu'il faut fournir ces informations pour remplir les fiches à renvoyer aux clients, les lacunes présentes dans l'écrit primaire sont généralement comblées sans que des incohérences puissent être identifiées au premier abord. Ceci montre que sont notées de façon préférentielle les informations qui servent dans les tâches de gestion et d'organisation des activités au sens large, tandis que les informations qui sont mobilisées dans le pilotage des cultures sont moins systématiquement relevées. 


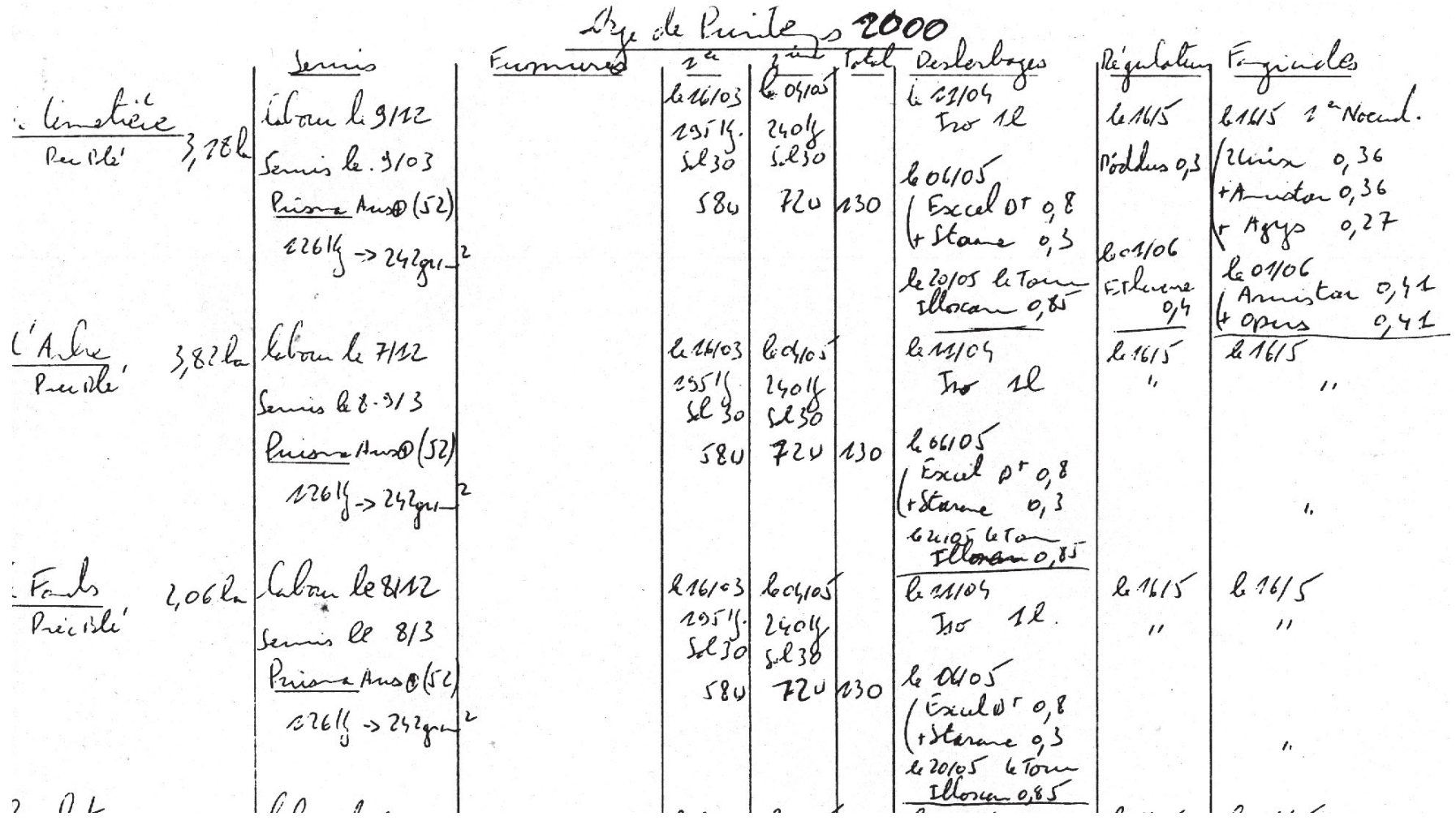

Fig. 2. Extrait d'une fiche par culture, reprenant les principales pratiques effectuées sur les nombreuses petites parcelles d'orge de l'exploitation (il y en a encore deux autres non présentées ici).

Tableau 4. Type de support primaire, personne inscrivant l'information, et informations notées par les agriculteurs.

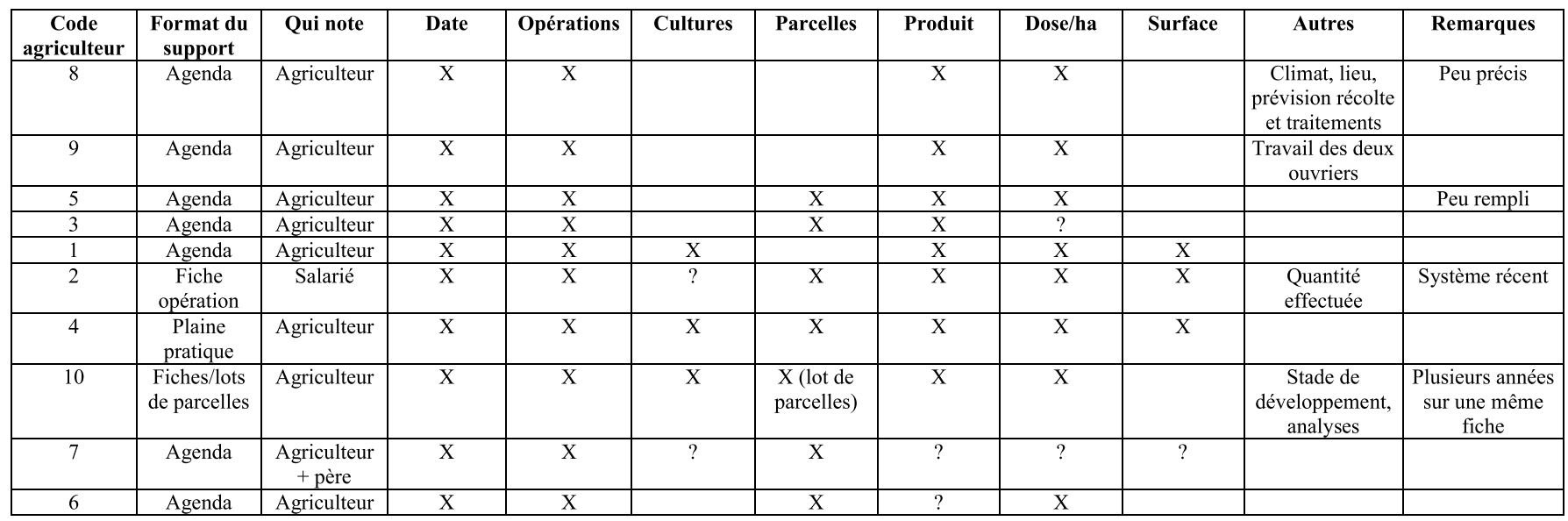

La diversité des pratiques de formalisation de l'information s'exprime donc à la fois dans le type de documents présents dans les exploitations (et dans ceux qui n'y sont pas), dans le format de stockage des informations et dans la façon dont sont construits les supports destinés aux clients. Nous pensons que cette diversité est pour partie liée à une différence entre exploitations quant à l'importance accordée à certaines activités de gestion de la production : planification des intrants, planification de l'utilisation annuelle ou pluriannuelle de la ressource terre, organisation du travail, pilotage des cultures.

\section{Adéquation des formats écrits des agriculteurs aux demandes externes}

Dans leur grande majorité, les firmes agroalimentaires demandent aux agriculteurs de remplir des «fiches par parcelle», appelées aussi fiches parcellaires, 
Tableau 5. Différentes stratégies d'ajustement des supports d'informations selon les contraintes organisationnelles.

\begin{tabular}{|c|c|c|c|}
\hline Exigences liées aux contrats & $\begin{array}{c}\text { Beaucoup de contrats exigeants en } \\
\text { informations et beaucoup de } \\
\text { clients différents }\end{array}$ & $\begin{array}{c}\text { Assez peu de contrats exigeants en } \\
\text { informations et un nombre moyen } \\
\text { de clients }\end{array}$ & $\begin{array}{c}\text { Peu de contrats exigeants en } \\
\text { informations et peu de clients } \\
\text { différents }\end{array}$ \\
\hline $\begin{array}{c}\text { Accumulation de contraintes sur } \\
\text { les assolements et systèmes de } \\
\text { culture }\end{array}$ & $1 \mathrm{~A}, 3 \mathrm{~L}$ & $1 \mathrm{~A}$ & $1 \mathrm{~A}$ \\
$(6)$ & $(6)$ & \\
\hline $\begin{array}{c}\text { Contraintes moyennes sur les } \\
\text { assolements et systemes de culture }\end{array}$ & $(8),(9)$ & $(3),(4),(10)$ & $2 \mathrm{~L}, 2 \mathrm{~L}$ \\
\hline $\begin{array}{c}\text { Peu de contraintes sur les } \\
\text { assolements et systèmes de culture }\end{array}$ & $1 \mathrm{~F}$ & & $(1),(2)$ \\
\hline
\end{tabular}

Cas 1 - Un seul support et pas de report : A agenda journalier ou F fiche par lots de parcelles ou carnet de plaine (données par parcelles)

Cas 2 - Deux reports et le second support est : F fiche par parcelle ou L logiciel

Cas 3- Trois reports et le troisième support est : A agenda journalier, ou F fiche par lots de parcelles, ou L logiciel

Présence d'un document d'assolement pluriannuel :

(x) code agriculteur

puisque c'est à cette échelle de la parcelle culturale qu'il est possible de reconstituer la succession des actions culturales qu'elles souhaitent confronter à leur cahier des charges. C'est ce format qui constitue, pour les firmes agro-alimentaires, le niveau privilégié de recueil de l'information. Cependant, la pertinence de cette entrée pour reconstituer les conditions de production d'une culture donnée est discutable pour plusieurs raisons.

Premièrement, les contours d'une parcelle culturale sont modifiés d'une année sur l'autre : deux parcelles voisines une année donnée, sont parfois regroupées l'année suivante, puis redécoupées ensuite selon d'autres limites que la première année ; il est donc difficile de reconstituer, avec la seule fiche parcellaire, l'histoire exacte de chacune de ces portions. Les échanges de parcelles, fréquents en Picardie en particulier chez les producteurs de pommes de terre pour respecter des délais de retour de la culture sur elle-même, compliquent encore la reconstitution historique sur plusieurs années des pratiques culturales; il est donc souvent nécessaire pour y arriver d'avoir recours à des données globales sur l'organisation de la production à l'échelle de l'exploitation qui n'existent pas dans les seules fiches parcellaires.

En second lieu, si la parcelle est pertinente comme unité d'action, elle ne l'est pas toujours comme unité de gestion; Cerf (1996), Maxime et al. (op. cit.), Aubry et al. (op. cit.) ont montré que selon que l'agriculteur planifie le travail, qu'il pilote les opérations techniques, qu'il cherche à contrôler la réalisation des opérations, l'unité spatiale de gestion est différente de la parcelle; ce peut être la sole (ensemble de parcelles consacrées, une année donnée, à la culture d'une même espèce), un lot de culture (ensemble de parcelles où une même espèce est cultivée de la même manière tout au long du cycle cultural). Il arrive que, pour des raisons de simplification du travail, des décisions prises sur une parcelle le soient en fonction d'observations faites sur d'autres (Aubry, 2000); ce sont là autant de justifications d'un recours à des informations sur l'organisation de la production ne figurant pas sur les fiches parcellaires.

Et enfin, les modalités actuelles de collecte et d'assemblage des produits agricoles par les entreprises de collecte-stockage (coopératives, négociants) consistent souvent à constituer des lots à partir de mélange des récoltes de plusieurs parcelles ou fractions de parcelles (Le Bail, 2000 ; Meynard et Le Bail, 2001). Dès lors, peuton justifier une forte exigence de traçabilité à la parcelle si cette exigence est absente au niveau de la constitution des mélanges?

Comme le montre le Tableau 1, les exploitations présentent des niveaux de contraintes variés en successions de cultures, de parcellaire, de débouchés commerciaux, de traçabilité. La prédominance de l'entité parcelle comme unité élémentaire de restitution des informations aux clients suggère que plus les parcelles sont nombreuses et les produits sous cahier des charges différents, plus la tâche d'enregistrement des informations est importante.

Ainsi, plus l'enregistrement est sollicité par les clients pour apporter des preuves de respect des cahiers des charges et des contrats, plus l'agriculteur devra, pour ce faire, enregistrer d'informations. Le Tableau 5, qui présente les documents utilisés par les agriculteurs au croisement des deux types de contraintes, montre qu'il n'en est rien. On constate que certains agriculteurs ayant beaucoup de contrats et de contraintes d'assolement se contentent d'une notation sur l'agenda structuré selon un format journalier ( ${ }^{\text {os }} 5$ et 8 ), tandis qu'à l'opposé, 
des agriculteurs ayant peu de contrats, peu ou pas de contraintes en matière d'assolement et de succession de cultures, opèrent un, voire deux reports pour compiler finalement leurs informations sur un logiciel selon un format parcellaire ( $\mathrm{n}^{\text {os }} 1$ et 2$)$.

Les enquêtes montrent donc qu'il existe une certaine déconnexion entre, d'un côté, les formats et supports adoptés par les agriculteurs pour mémoriser par écrit des informations, et de l'autre, la pression exercée par l'aval pour une structuration de ces informations parcelle par parcelle. Elles montrent aussi qu'au-delà d'une certaine complexité, du parcellaire surtout, une structuration des informations à la parcelle devient alors trop contraignante au regard de l'intérêt de ces informations pour l'agriculteur. Il est alors rare qu'un relevé par parcelle des informations soit conservé ou reporté sur informatique.

En revanche, un document de gestion interne semble très souvent associé aux situations à forte pression contractuelle : le document d'assolement pluriannuel (Tab. 5). En effet, il semble que ce dernier permette, en particulier, de rassurer le client sur le passé de la parcelle (précédents culturaux déconseillés, épandages de boues...) et vienne compléter les informations portées par exemple sur l'agenda structuré selon la date. Il constitue même pour certaines exploitations la seule source d'information en fin de saison pour remplir les fiches parcellaires demandées par les clients (Encadré 2).

\section{Des aides à la mémorisation, reflets de l'organisation interne de l'exploitation}

La nature et la quantité d'informations à mémoriser (par un écrit ou en mémoire interne) par les agriculteurs sont donc liées à l'organisation interne de leurs activités. Ainsi, sont généralement notées les informations qui servent dans les tâches de gestion et d'organisation des activités au sens large (affectation des hommes et des machines dans le temps et l'espace, affectation des opérations à des hommes, contrôle des délais de réalisation, contrôle des quantités des intrants les plus chers... ), tandis que les informations qui sont mobilisées dans le pilotage des cultures sont moins systématiquement relevées.

La quantité d'informations à mémoriser peut être en effet assez limitée dès que les situations productives sont homogènes : de grandes parcelles en petit nombre avec peu de cultures différentes. Dans ce cas, structurer les informations par culture suffit à retrouver les informations par parcelle et à remplir les fiches demandées par les clients. Dans d'autres cas, le milieu physique, l'histoire et les stratégies commerciales de l'exploitation complexifient les combinaisons productives et les informations nécessaires à mémoriser pour les gérer. Deux stratégies d'adaptation pour réduire la quantité des informations à mémoriser et simplifier les écrits transparaissent dans les exploitations enquêtées. Soit l'agriculteur réduit la complexité de son assolement, soit il simplifie les modes de saisie et de stockage de ses informations. Ainsi, l'agriculteur 10, qui a un parcellaire morcelé et dispersé, n'a pas de document d'assolement pluriannuel et note les informations sur des fiches par lots de parcelles. Cette notation lui suffit car il a groupé ses parcelles en trois zones à chacune desquelles il affecte une rotation triennale stable : ainsi la présence d'une culture sur une zone permet de connaître quasi automatiquement la culture précédente et celle qui suivra. Le format choisi par l'agriculteur est donc associé à la façon dont il se représente l'organisation spatiale de son exploitation, mais aussi l'organisation de ses activités productives dans le temps ou dans la répartition du travail. De son côté, l'agriculteur 3 conserve les informations par espèce cultivée, en établissant, quand il réalise un report des informations du support primaire sur le support secondaire, une fiche pour toutes les parcelles consacrées à cette espèce. Cette stratégie de simplification des modes de saisie et de stockage des informations peut se comprendre compte tenu du nombre important de ses parcelles.

Ces deux exemples illustrent les relations qui peuvent exister entre les formats et les pratiques de report des agriculteurs et le degré de complexité organisationnelle que l'agriculteur a à gérer. L'intérêt d'une saisie à la parcelle n'a de sens que dans les exploitations agricoles où le nombre de parcelles permet d'avoir un nombre suffisamment petit de parcelles homogènes, tous critères confondus, pour être pris en compte. Dans le cas contraire, l'agriculteur simplifie ses enregistrements. Pour pouvoir juger de la conformité à un cahier des charges des pratiques culturales appliquées à une parcelle donnée, il faut alors tabler sur la capacité de l'agriculteur à reconstituer ses règles d'organisation de la production dans l'exploitation, notamment celles concernant l'assolement et les diverses successions de cultures, ainsi que l'organisation du travail (Aubry et al., op. cit. ; Papy, op. cit.). Les contraintes informationnelles dans l'organisation des activités de l'exploitation expliquent la diversité que nous avons mise en évidence des réponses des agriculteurs aux demandes d'informations portées par les cahiers des charges de leurs clients.

\section{Rapprocher mémoire et preuve : suggestions de stratégies}

Nous avons donc enregistré une diversité des documents de saisie des informations par les agriculteurs, mais aussi l'existence d'un décalage entre les formats demandés par leurs clients et ceux que produisent les agriculteurs pour eux-mêmes. Le rapprochement de ces deux points de vue suppose alors un arbitrage, car pour 
Tableau 6. Le format entre connaissance des exploitations et harmonisation des informations.

\begin{tabular}{|c|c|c|c|}
\hline & \multicolumn{2}{|c|}{ Demande d'harmonisation des informations } \\
\hline & & Faible & Forte \\
\hline \multirow{2}{*}{$\begin{array}{l}\text { Besoin de } \\
\text { connaissance du } \\
\text { fonctionnement } \\
\text { global de } \\
\text { l'exploitation }\end{array}$} & Faible & $\begin{array}{c}\text { Cas } 2 \\
\text { Formats actuels de saisie } \\
\text { l'agriculteur }\end{array}$ & $\begin{array}{c}\text { Cas 1 } \\
\begin{array}{c}\text { Fiches simplifiées des } \\
\text { industriels }\end{array}\end{array}$ \\
\hline & Fort & $\begin{array}{c}\text { Cas } 3 \\
\text { Autres représentations du } \\
\text { fonctionnement global de } \\
\text { l'EA }\end{array}$ & $\begin{array}{c}\text { Cas } 4 \\
\text { ISO } 9000 \\
\text { (harmonisation par le } \\
\text { système qualité) }\end{array}$ \\
\hline
\end{tabular}

prendre en compte cette diversité, il faut être au plus près de la gestion organisationnelle des agriculteurs. La qualité des informations, mais aussi leur simplification permettent d'obtenir à un moindre coût les garanties recherchées par les entreprises (contrôle du contrat, informations à transmettre aux consommateurs. . .).

\section{Transférer ou ne pas transférer l'information}

Actuellement, comme nous l'avons déjà dit, pour la plupart des firmes agro-alimentaires, la question de la formalisation des façons de produire des agriculteurs se pose essentiellement en termes d'amélioration des transferts d'informations entre les agriculteurs et leurs divers interlocuteurs. Implicitement, la transmission des informations générées par les agriculteurs vers leurs clients est considérée comme incontournable pour permettre la traçabilité.

En réalité, le transfert d'informations n'est indispensable pour les firmes que s'il est associé au suivi de production et à l'organisation de leur approvisionnement. Certes, les firmes sont elles-mêmes soumises à des exigences de traçabilité qui justifient leurs demandes auprès des agriculteurs, mais les écrits de traçabilité ont rarement une utilité directe en termes de gestion de la production pour les entreprises alors même que leur recueil a un coût pour l'entreprise comme pour l'agriculteur. Ainsi, ces informations écrites sont en fait souvent complétées pour assurer la gestion de la production : ex ante, un entretien initial est réalisé, visant à évaluer la capacité de l'agriculteur à respecter a priori le cahier des charges techniques ; ex post, des visites ou des observations sont réalisées par les techniciens de l'entreprise pour le suivi des cultures et l'ajustement du plan d'approvisionnement. Ainsi, les informations nécessaires aux clients combinent, selon les cas, des enregistrements à la parcelle, mais aussi une connaissance plus globale du fonctionnement de l'exploitation agricole.

Dès lors, il ne s'agit pas seulement, et peut-être pas essentiellement, de résoudre la question du transfert des informations des exploitations vers leurs clients. Il s'agit de concevoir des stratégies de formalisation des manières de produire qui, à moindre coût, permettent d'apporter des garanties sur ces dernières et donnent aux agriculteurs et à leurs clients les moyens de disposer des informations utiles à leurs activités gestionnaires respectives.

À la différence de certains travaux engagés aujourd'hui autour de la simplification et de l'informatisation d'un document unique (la fiche parcellaire type), notre analyse conduit à souligner la nécessité de maintenir cette diversité, justifiée par l'existence d'usages et de traitements propres à chaque agriculteur pour sa gestion interne et à explorer des solutions d'harmonisation entre fourniture et demande d'informations.

\section{Plusieurs stratégies d'harmonisation possibles}

Pour réaliser le nécessaire arbitrage entre la reconnaissance de la diversité des documents élaborés individuellement par les agriculteurs et l'harmonisation, à un niveau collectif, des informations pour leurs clients, nous proposons quatre solutions possibles, non exclusives les unes des autres (Tab. 6).

La première (cas 1), souvent évoquée actuellement, consiste en un simple ajustement des formats de saisie de l'information (harmonisation et simplification des fiches parcellaires...) et est accompagnée de solutions informatiques pour la saisie et le stockage de ces informations au champ afin de réduire les reports et minimiser les saisies. L'effort d'harmonisation, notamment au niveau de groupes de normalisation, porte aujourd'hui surtout sur les formats de saisie proposés par les clients des agriculteurs (Martin et Pagès, 2002). La codification par parcelle demeure ici le format de stockage de l'information privilégié, car elle facilite la recherche des informations contenues dans l'engagement de production (localisation, surface, précédent cultural). Cette solution suppose une diffusion de l'informatique en milieu agricole, pourtant encore très limitée aujourd'hui. Un tiers des exploitations seraient équipées d'après le dernier recensement agricole, mais seulement 10 à $15 \%$ en auraient un véritable usage professionnel pour la gestion technique ou économique. 
La seconde (cas 2) concerne plutôt les situations où la nécessité $d$ 'harmoniser les formats d'information est faible, soit parce qu'il s'agit de productions spécifiques impliquant peu d'agriculteurs permettant un traitement plus aisé de l'information pour leurs clients, soit parce que ces mêmes clients n'ont pas besoin d'avoir une connaissance précise du fonctionnement de l'exploitation agricole pour évaluer la capacité de l'agriculteur à respecter ses engagements contractuels. Dans ce cas, les documents élaborés par l'agriculteur selon ses propres formats peuvent suffire.

La troisième (cas 3) repose sur d'autres représentations $\mathrm{du}$ fonctionnement global de l'exploitation agricole. Elle consiste à formaliser les processus de planification des activités productives afin d'aider les agriculteurs à raisonner leurs marges de manœuvre. Il leur faut répondre par exemple à de nouvelles exigences de la part de leurs clients ou se porter vers de nouveaux débouchés et fournir une preuve ex ante aux clients. En effet, des travaux antérieurs (Cerf, op. cit. ; Aubry et al., op. cit. ; Maxime et al., op. cit.) ont montré qu'il est possible de faire expliciter à l'agriculteur la façon dont il raisonne l'affectation de ses terres et de ses ressources en matériel et en main-d'œuvre, ainsi que les ajustements qu'il envisage en fonction de caractéristiques des situations productives qu'il est capable d'anticiper.

La quatrième (cas 4), quoique peu développée encore en agriculture, constitue une voie intermédiaire dans laquelle la certification globale du système (par une adaptation du modèle ISO $9000^{2}$ ) permet de garantir le respect des cahiers des charges et de la traçabilité. Cette solution peut se substituer à une transmission systématique de toutes les informations. Le niveau de généralité des formats normalisés, notamment le manuel qualité ${ }^{3}$, permet une prise en considération de la diversité des «écrits pour soi » élaborés par les agriculteurs. Plusieurs initiatives récentes ont permis d'adapter ces normes au contexte de l'agriculture (Mazé et al., 2002).

Ces quatre solutions ne sont évidemment pas équivalentes du point de vue des exigences de mise en forme des informations par les agriculteurs, des compétences nécessaires pour mettre en place et contrôler de nouveaux modes de formalisation, et des relations que cela implique entre les agriculteurs et leurs clients. Dans certains cas, il peut être plus simple de remplir une fiche

\footnotetext{
${ }^{2}$ Les normes de la série ISO 9000 définissent des modèles types de système qualité pour les certifications d'entreprise et pour l'assurance qualité dans le cadre des relations clientsfournisseurs.

${ }^{3}$ Format principal de la norme ISO, il est «l'image écrite de l'organisme en matière de politique qualité et d'organisation mise en place pour respecter cette politique. C'est le document sur lequel s'appuient tous les autres documents concernant la qualité ». (Froman, 1995.)
}

parcellaire type que d'avoir à expliciter, puis formaliser le fonctionnement global de son exploitation agricole.

Si l'on peut penser qu'un travail de formalisation supplémentaire demandé à l'agriculteur présente une certaine utilité interne, dès lors que ce dernier recherche des débouchés plus qualifiés, en revanche, il faut s'interroger sur l'utilité effective pour les entreprises agroalimentaires de ces formalisations. Ces solutions nécessitent une négociation entre les agriculteurs et leurs clients et, le cas échéant, une réorganisation de leurs relations contractuelles. De plus, se pose la question de savoir sous quelles conditions il est opportun pour l'agriculteur d'exposer ainsi ce qui constitue son savoir-faire.

\section{Conclusion}

Cette étude empirique a montré qu'il n'y a pas de liaison simple entre le nombre de cahiers des charges ou de demandes d'informations de la part des clients et les documents utilisés par les agriculteurs pour saisir et stocker les informations. Ceci tient au fait que les agriculteurs notent des informations pour leurs besoins propres de gestion interne et que leurs formats de saisie des informations ne sont pas indépendants des contraintes organisationnelles et de la façon dont ils les gèrent. L'ensemble des documents élaborés par les agriculteurs fait «système » : il forme un tout cohérent et complémentaire de l'organisation propre de chaque exploitation. Dès lors, les solutions proposées aux agriculteurs pour faire évoluer leurs pratiques de gestion des informations doivent tenir compte de la situation spécifique de chaque exploitation.

Face à ces résultats, plusieurs stratégies sont possibles selon les pratiques des agriculteurs et leurs engagements contractuels. De nouvelles façons de formaliser les manières de produire peuvent s'avérer nécessaires lorsque les agriculteurs comme leurs clients souhaitent repositionner les informations au sein d'une évaluation globale $\mathrm{du}$ fonctionnement de l'exploitation agricole et en particulier des modes d'affectation des ressources. La reprise des documents élaborés par l'agriculteur pour sa propre gestion des ressources dans le cadre d'un manuel qualité (norme ISO) ou la formalisation des raisonnements d'affectation des ressources (Papy, 2001) peuvent constituer les supports adéquats à l'acquisition d'une représentation partagée de ce fonctionnement par les acteurs impliqués.

Cependant, l'agriculteur réalise rarement seul une démarche de formalisation qui peut être facilitée par le recours à un tiers. Le rôle du tiers est généralement assumé dans l'industrie par le « responsable qualité ». Les conseillers (de chambres d'agriculture et de centres de gestion) qui gravitent aujourd'hui autour des agriculteurs, les agents de plaine ou techniciens des entreprises agro-alimentaires, sont-ils disponibles et ont-ils les 
compétences pour rentrer dans ce jeu d'explicitation et de formalisation et, le cas échéant, pour l'aider à valoriser ses propres marges de manœuvre et mieux s'adapter à des cahiers des charges? Les entreprises agro-alimentaires sont-elles prêtes à payer le prix de la compétence?

\section{Références}

Ackerman, M.S., Malone, T.W., 1990. Answer garden : A tool for growing organisational memory, Proceedings of ACM Conference on Office Information Systems, ACM Press, 31-39.

Aoki, M., 1986. Horizontal versus Vertical Information Structure of the Firm, American Economic Review, 2, 971-983.

Aubry, C., Biarnès, A., Maxime, F., Papy, F., 1998. Modélisation de l'organisation technique de la production dans l'exploitation agricole, in Brossier, J., Dent, B., (Eds), Gestion des exploitations et des ressources rurales. Entreprendre, négocier, évaluer. Farm and Rural Management. New contents, new contraints, new opportuntie, Versailles, INRA SAD, 25-43.

Aubry, C., 2000. Une modélisation de la gestion de production l'exploitation agricole, Revue française de gestion, 129, 32-46.

Barzel, Y., 1977. Some fallacies in the interpretation of informations costs, Journal of Law and Economics, 20, 2, 291-307.

Cerf, M., 1996. Approches cognitives des pratiques agricoles : intérêts et limites pour les agronomes, Natures Sciences Sociétés, 4, 4, 327-339.

Cochoy, F., Garel, J.P., de Terssac, G., 1998. Comment l'écrit travaille l'organisation : le cas des normes ISO 9000, Revue française de sociologie, 39, 4, 673-699.

Fabbe-Costes, N., 1997. Les multiples rôles du système d'information et de communication dans l'action stratégique, in Avenier, M.J. (Ed.), La Stratégie "chemin faisant ", Paris, Economica, 189-215.

Froman, B., 1995. Le Manuel Qualité : outil stratégique d'une démarche qualité. Afnor.

Fraenkel, B., 1995. La traçabilité, une fonction caractéristique des écrits de travail, Connexion, 65, 1, 63-75.

Grosjean, M., Lacoste, M., 1998. L'oral et l'écrit dans les communications de travail ou les illusions du «tout écrit», Sociologie du travail, 4, 439-461.
Joly, N., 1997. Écritures du travail et savoirs paysans. Aperçu historique et lecture de pratiques. Les agendas des agriculteurs. Thèse de doctorat, Université Paris X, Nanterre.

Le Bail, M., 2000. Qualité des produits végétaux et territoire : contribution de l'agronomie, OCL, 7, 499-503.

Lorenz, E., 2001. Models of cognition, the contextualization of knowledge and organizational theory, Journal of Management and Governance, 5, 307-330.

Martin, C., Pagès, C., 2002. Conditions et moyens d'une gestion informatisée des données pour la mise en œuvre de l'agriculture raisonnée, Ingénieries Eau-Agriculture-Territoires, 30, 43-58.

Maxime, F., Nicoletti, J.P., Leroy, P., Papy, F., 1997. Donner de la souplesse au choix d'assolement par des rotations cadres, in Aide à la décision et choix de stratégies dans les exploitations agricoles, actes du colloque de Laon, 10-11 /12/1996, INRA, Le Biopôle, 85-99.

Mazé, A., Galan, M.B., Papy, F., 2002. The Governance of Quality and Environmental Management Systems in Agriculture : New Challenges and Research Issues, in Hagedorn, K., Environmental Co-Operation and Institutional Change. Cheltenham, Edward Elgar, 162-182.

Meynard, J.-M., Le Bail, M., 2001. Isolement des collectes et mâेtrise des disséminations au champ, Programme de recherche «Pertinence économique et faisabilité d'une filière sans utilisation d'OGM », Paris, INRA, INA P-G.

Ognov, A., 2000. Systèmes d'information et traçabilité dans les exploitations agricoles. Éléments de diagnostic. Mémoire de Diplôme d'agronomie approfondi INA P-G, Paris.

Ognov, A., 2001. Représentation de l'organisation de l'exploitation - Maîtrise de la production, Paris, Document de travail AgroTransfert Picardie / INRA SAD-APT.

Owen, D., 1986. Answer first, then questions, in Norman, D.A., and Draper, S.W. (Eds), User Centred System Design, Reix Hillsdale, N.J. : Lawrence Erlbaum Associates.

Paillotin, G., 2000. L'Agriculture raisonnée. Rapport au ministre de l'Agriculture et de la Pêche, http://www.agriculture.gouv.fr

Papy, F., 2001. Interdépendance des systèmes de culture dans l'exploitation, in Malézieux, E, Trébuil, G., et Jaeger, M. (Ed.), Modélisation des agro-écosystèmes et aide à la décision, Paris, CIRAD-INRA, 51-74.

Radner, R., 1993. The organization of decentralized information processing, Econometrica, 61, 5, 1109-1146.

Reix, R., 1996. Systèmes d'information et management des organisations, Paris, Vuibert.

Reçu le 17 avril 2003. Accepté le 4 décembre 2003.

$\overline{\text { To access this journal online: }}$ www.edpsciences.org 